\title{
Non-Cooperative Power Control for Faded Wireless Ad Hoc Networks
}

\author{
Xiaolu Zhang, Meixia Tao and Chun Sum Ng \\ Department of Electrical and Computer Engineering \\ National University of Singapore \\ Singapore 117576 \\ Email: $\{$ zhangxiaolu, mxtao, elengcs $\} @$ nus.edu.sg.
}

\begin{abstract}
The problem of non-cooperative power control is studied for wireless ad hoc networks supporting data services. We develop a maximum throughput based non-cooperative power control game (MT-NPG), where each transmitter node requires only the effective interference measurement from its corresponding receiver. An expression for the optimal power control to maximize the average transmission rate for each individual link is derived. Then, we design a distributed iterative power updating algorithm to approximate the optimal solution. The existence and uniqueness of Nash equilibrium for the proposed game is investigated. Simulation results show that the proposed MT-NPG yields a significant improvement in the sum of average achievable rates when compared with existing approaches.
\end{abstract}

\section{INTRODUCTION}

Power control is a basic technique of resource management to mitigate interference and improve system performance. For traditional voice and other delay-constrained applications, power control can be viewed as a method to compensate the fluctuation of wireless channels [1] and [2].

However, this is not true for "elastic" data applications. Since data applications can tolerate a larger delay, it is possible to adapt the transmission parameters, such as transmission rate and transmission power, to the underlying channel, interference, and system preferences to maximize the throughput [3]. In a single-user fading channel, the transmission scheme that maximizes the long-term throughput results in transmitting more power when the channel conditions are favorable and vice versa [4]. The power control mechanisms include also user scheduling when considering the network aspects as well as link performance. Power control in multiple access wireless data networks is studied in [5] and [6], where a centralized controller is required to collect users' information and schedule their transmission power and rate. In wireless ad hoc networks, however, there is no centralized control unit such as a base station (BS) or an access point (AP). Thus, scheduling becomes a very complex and challenging task, especially when there are a large number of user nodes and a diversity of applications.

To date, the game theory is shown to be a useful tool to model the resource allocation problem in wireless ad hoc networks as a non-cooperative game. An important assumption in game theory is that the users are rational and selfish. The best response of each user to the actions taken by others is to maximize its own utility [7]-[10]. In [8], Saraydar et al. resort to pricing to guide non-cooperative users for more efficient allocation of resources. In [7], games at both the user level and the system level are designed. Unfortunately, these games involve the participant of $\mathrm{BS}$, and determining the pricing function and utility function is non-trivial. Finding the "optimum" pricing and utility function is still an open problem. In [11], Sung et al. propose an opportunistic power algorithm (SOPA) which aims at maintaining a constant signalinterference product. This algorithm achieves considerable improvements in system throughput over the Foschini-Miljanic algorithm for data service. Yet, it may not be throughput optimal. In addition, SOPA is based on instantaneous channel state information, and thus a large amount of overhead is involved in channel feedback. Moreover, when the channel varies rapidly, the convergence of SOPA is not guaranteed.

This paper considers a practical algorithm of noncooperative power control in wireless ad hoc networks. The long-term throughput of each user is to be maximized under a given time-average power constraint. This time-average constraint allows us to implement opportunistic communication [5]. That is, more power is used for transmission when interference is low, and vice versa. We consider a timevarying channel formed by large-scale fading and small-scale multipath fading components. It is assumed that the largescale fading remains constant within the time scale of the application of the power control algorithm. Using this channel model, we first derive an optimal power control strategy that maximizes the throughput of individual links when only effective interference (defined in Section II) is known. The proposed optimal power control strategy implicitly includes an admission control: when the effective interference surpasses a certain threshold, the transmission power is cut off. We then construct an iterative function for updating power levels. This function belongs to a type-II standard function defined by Sung et at in [11]. It can be proven that the proposed iterative procedure converges to a unique fixed point. Another contribution of this paper is the proposal of a novel method to guarantee that the power consumption always satisfies the average power constraint. The power control structure consists of an outer loop algorithm that adjusts system parameters and an inner loop which calculates optimal power levels based on effective interference measurements. Simulation results show that the proposed MT-NPG yields a significant improvement 
in the sum of average achievable rates when compared with existing approaches.

\section{System Model}

We consider a wireless data network with a set of $N$ communication links, denoted as $\mathcal{N}=\{1,2, \ldots, N\}$. Link $i$ contains a transmitter node $T X_{i}$ and a desired receiver node $R X_{i}$. For each link, the transmission power is determined by the channel condition and the interference level so as to maximize the long-term throughput. The time-varying channel gain from $T X_{j}$ to $R X_{i}$ is modelled as $c_{i j}(t)=g_{i j}(t) h_{i j}(t)$, where $g_{i j}$ is the large-scale fading component including both path loss and shadowing, and $h_{i j}$ is the normalized smallscale multipath fading component, which follows exponential distribution in Rayleigh fading channels considered in this paper. The multipath fading is assumed to fluctuate rapidly that the transmitter node cannot capture the instantaneous value of $h_{i j}$. The transmission power can thus only be updated at a period greater than the channel coherence time. On the other hand, the large-scale fading $g_{i j}$ changes slowly and remains approximately constant within each period of power update. Here, we define one period of power update as one time slot. These above channel assumptions hold in general wireless mobile environments.

The information transmission rate is determined by a certain rate-power function. Because communication rates over the link may be influenced by interference from other links, achievable transmission rate in a time slot could be approximated by using signal-to-interference-plus-noise ratio (SINR). Let $p_{i}$ denote the transmission power of $T X_{i}, \eta_{i}$ denotes noise power observed at $R X_{i}$, and $\theta_{i j}$ represents the normalized cross-correlation between the waveforms of $T X_{i}$ and $T X_{j}$. To keep the notation clear, the power gain is redefined to incorporate the cross-correlations:

$$
g_{i j}:=\theta_{i j} g_{i j} .
$$

We define effective interference, $I_{i}$, observed by $R X_{i}$ after averaging over small-scale fading as

$$
I_{i}=\frac{1}{g_{i i}}\left(\sum_{j \neq i} g_{i j} p_{j}+\eta_{i}\right) .
$$

When characterizing the achievable rate as mutual information, we can express the achievable rate as

$$
\begin{aligned}
& R_{i}\left(p_{i}, I_{i}\right) \\
= & E_{\mathbf{h}_{i}}\left\{\ln \left[1+\frac{g_{i i} h_{i i} p_{i}}{\sum_{j \neq i} g_{i j} h_{i j} p_{j}+\eta_{i}}\right]\right\} \\
\geq & E_{h_{i i}}\left\{\ln \left[1+\frac{h_{i i} p_{i}\left(I_{i}\right)}{I_{i}}\right]\right\} \\
= & e^{x} \int_{x}^{\infty} \frac{e^{-t}}{t} \mathrm{~d} t,
\end{aligned}
$$

In (2), $\mathbf{h}_{i}:=\left[h_{i 1}, h_{i 2}, \ldots, h_{i N}\right]$, and in (4), $x:=\frac{I_{i}}{p_{i}\left(I_{i}\right)}$. Because the elements in $\mathbf{h}_{i}$ are independent, we can take the expectation over each $h_{i j}$ separately in (2).
The network considered is a distributed system with no centralized coordinator. Thus, the effective interference $I_{i}$ is the only information available at $T X_{i}$, which can be obtained via a feedback channel from $R X_{i}$. The power $p_{i}$ can be adjusted according to the effective interference so as to maximize the average achievable rate of link $i$. Here the average achievable transmission rate of link $i$ is defined as the expected conditional achievable transmission rate over the probability density function (pdf) $f\left(I_{i}\right)$, and is given by

$$
\bar{R}_{i}:=\mathbb{E}_{I_{i}}\left[R_{i}\left(p_{i}, I_{i}\right)\right]=\int_{0}^{\infty} R_{i}\left(p_{i}\left(I_{i}\right), I_{i}\right) f\left(I_{i}\right) \mathrm{d} I_{i} .
$$

\section{Maximum Throughrut Non-CoOperative Power Control GAme (MT-NPG)}

In a distributed wireless network, it is impractical to collect all individuals' information to reach an operating point that optimizes the resource allocation due to the large signaling overhead. Each node tries to maximize its performance under average power constraint as a selfish individual. We take the average achievable rate defined in (5) as the performance metric, i.e., each node competes with other nodes to maximize its own average achievable rate by adjusting power according to its effective interference information.

Let $G=\left[\mathcal{N},\left\{p_{i}\right\},\left\{\bar{R}_{i}\right\}\right]$ denote the non-cooperative power control game, and $\mathbf{p}:=\left[p_{1}, p_{2}, \ldots, p_{N}\right]$ denote the power level selected according to the effective interference information in the NPG. The resulting average achievable rate for link $i$ is $\bar{R}_{i}=\mathbb{E}\left[R_{i}(\mathbf{p})\right]$, and an alternative notation is $\mathbb{E}\left[R_{i}\left(p_{i}, \mathbf{p}_{-i}\right)\right]$, where $\mathbf{p}_{-i}$ denotes the vector consisting of elements of $\mathbf{p}$ other than the $i^{\text {th }}$ element. Because the average achievable rate $R_{i}$ is a function of $I_{i}$, and $I_{i}$ is a function of $\mathbf{p}_{-i}$, the $i^{t h}$ node's average achievable rate does not depend on $p_{i}$ only, but also on the choice of other nodes' power.

We assume the average power constraint of $T X_{i}$ is $\bar{p}_{i}$. Each transmitter node can adjust its power to the effective interference $I_{i}\left(\mathbf{p}_{-i}\right)$ so as to maximize its own average achievable rate. Node $T X_{i}$ 's best response for a given $I_{i}$ can thus be expressed as

$$
p_{i}^{*}:=F_{i}\left(I_{i}\right)=\arg \max _{\mathbb{E}\left[p_{i}\left(I_{i}\right)\right] \leq \bar{p}_{i}} \bar{R}_{i} .
$$

\section{A. Optimal power control}

In this subsection, we derive the optimal power $p_{i}^{*}$. The optimization problem is expressed as, for all $i \in \mathcal{N}$,

$$
\begin{array}{cc}
\max _{p_{i}} & \bar{R}_{i}, \\
\text { s.t. } & E\left[p_{i}\left(I_{i}\right)\right] \leq \bar{p}_{i} \\
& p_{i}\left(I_{i}\right) \geq 0,
\end{array}
$$

By using Lagrange-multiplier based constraint optimization, the augmental integral can be formulated as

$$
L_{i}=\int_{I_{i}}\left[R_{i}\left(p_{i}\left(I_{i}\right), I_{i}\right)-\lambda_{i} p_{i}\left(I_{i}\right)\right] f\left(I_{i}\right) \mathrm{d} I_{i},
$$

where $\lambda_{i}$ is the Lagrange multiplier, independent of $I_{i}$. It can be shown that $R_{i}$ is a concave function of $p_{i}$. Hence, EulerLagrange equation is the necessary and sufficient condition of 


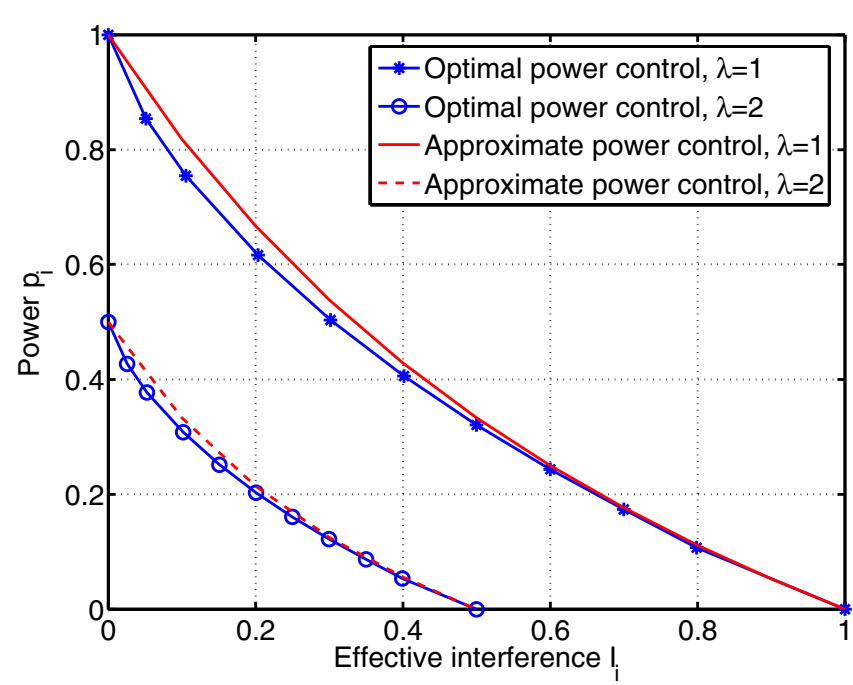

Fig. 1. Best-response functions for the optimal and approximate power control with $\lambda=1$ and $\lambda=2$ respectively.

this optimization problem [12]. Application of Euler-Lagrange equation results in

$$
p_{i}^{*}=\frac{1}{\lambda_{i}}\left(1-x e^{x} \int_{x}^{\infty} \frac{e^{-t}}{t} \mathrm{~d} t\right),
$$

where $x=\frac{I_{i}}{p_{i}^{*}\left(I_{i}\right)}$. Similar to [13], it can be proven that $p_{i}$ has a unique positive fixed-point solution $F_{i}\left(I_{i}\right)$ for any $I_{i}<\frac{1}{\lambda_{i}}$.

Some examples of the power control function $p_{i}^{*}$ are shown in Fig. 1 (the approximate one in the figure will be discussed in Section III-B). It is observed that the optimal power $p_{i}^{*}$ is a decreasing convex function of $I_{i}$. Moreover, when $I_{i}$ is above the threshold $1 / \lambda_{i}$, the optimal scheme will cut off the transmission power. Thus, our solution implicitly includes an admission control. This phenomenon is similar to the well-known water-filling policy. The parameter $\lambda_{i}$ can be determined by the average power constraint $\bar{p}_{i}$ (6). Conversely, we can adjust the value of $\lambda_{i}$ so as to guarantee a given longterm power constraint. For instance, diminishing long-term power consumption can be realized by increasing the value of $\lambda$. In addition, it is observed from Fig. 1 that the optimal power allocation is upper bounded by $1 / \lambda$, which is suitable for realization in practice since mobile terminals, in general, have a limited peak power.

While each transmitter node adjusts the transmission power to maximize the average achievable rate, it contributes to the interference affecting other nodes. The interaction among each other results in a power control game. Starting from $\mathbf{p}^{(0)}$, we update the power vector in a round-robin fashion. Like the Yate's framework [14], the power vector is updated by an iterative function

$$
\mathbf{p}^{(n+1)}=\mathbf{F}\left(\mathbf{p}^{(n)}\right),
$$

where $\mathbf{F}(\mathbf{p})=\left(F_{1}\left(\mathbf{p}_{-1}\right), \ldots, F_{N}\left(\mathbf{p}_{-N}\right)\right)$, and each $F_{i}(\cdot)$ can be obtained from the optimal solution (7).
Proposition 1: The iterative function $\mathbf{F}(\cdot)$ in (8) that achieves the optimal solution (7) is neither standard nor type-II standard [11].

Hence, $\mathbf{F}(\cdot)$ cannot guarantee the convergence of the power vector $\mathbf{p}^{(n)}$ to a fixed point.

\section{B. Iterative function construction}

In this subsection, we construct a novel iterative power update function $\mathbf{J}(\mathbf{P})$ with guaranteed convergence. To achieve a near-optimal performance, the iterative function is required to resemble the features of the optimal solution $F_{i}(\cdot)$ derived from (7). Specifically, the iterative function $J_{i}\left(I_{i}\right)$ should include the limiting points $\left(\frac{1}{\lambda_{i}}, 0\right)$ and $\left(0, \frac{1}{\lambda_{i}}\right)$. It should also be a decreasing and convex function of $I_{i}$.

To facilitate the construction of iterative function with the above constraints, we approximate the solution to equation (8) using

$$
p_{i}=\min \left[\left(\frac{2}{\lambda_{i}^{2} I_{i}+\lambda_{i}}-\frac{1}{\lambda_{i}}\right)^{+}, \frac{1}{\lambda_{i}}\right] \text {, }
$$

where $(x)^{+} \triangleq \max (0, x)$. The best-response function examples for this approximate power control are shown in Fig. 1.

We now illustrate how this heuristic approximation leads to an iterative algorithm that converges. Define a new variable $q_{i}$, and let

$$
q_{i}=J_{i}\left(\mathbf{q}_{-i}\right)=\frac{2}{\lambda_{i}^{2} I_{i}+\lambda_{i}} .
$$

Hence,

$$
p_{i}=\min \left[\left(q_{i}-\frac{1}{\lambda_{i}}\right)^{+}, \frac{1}{\lambda_{i}}\right] .
$$

The vector $\mathbf{q}$ is updated by the iterative function

$$
\mathbf{q}^{(n+1)}=\mathbf{J}\left(\mathbf{q}^{(n)}\right) .
$$

We prove in the appendix that the iterative function is Type-II standard, i.e., J satisfies the following two conditions [11],

1) Type-II Monotonicity: If $\mathbf{q} \leq \mathbf{q}^{\prime}$, then $\mathbf{J}(\mathbf{q}) \geq \mathbf{J}\left(\mathbf{q}^{\prime}\right)$.

2) Type-II Scalability: For all $\alpha>1, \mathbf{J}(\alpha \mathbf{q})>\left(\frac{1}{\alpha}\right) \mathbf{J}(\mathbf{q})$. when the inequality

$$
\frac{\eta_{i}}{g_{i i}}+\frac{1}{\lambda_{i}}-\sum_{i \neq j} \frac{g_{i j}}{g_{i i} \lambda_{j}}>0
$$

is satisfied. Note that this condition holds owing to the fact that $g_{i j}$ is much less than $g_{i i}$ in practical systems.

The outline of the proposed iterative power update for each node is as follows:

1) Set initial values

$$
\text { Let } \mathbf{p}^{(0)}=\mathbf{p}_{\max }, \lambda_{i}^{(0)}=\frac{1}{p_{\max }}
$$

2) Long-term update every $M$ time slots $(M \geq 50)$

Measure the actual amount of average power consumption $\bar{p}_{i}{ }^{\prime}$ in $M$ time slots, and compare it with average power constraint $\bar{p}_{i}$. Update $\lambda_{i}$ according to $\lambda_{i}^{(n+1)}=\lambda_{i}^{(n)}+\Delta\left(\bar{p}_{i}{ }^{\prime}-\bar{p}_{i}\right)$, where $\Delta$ is the step size.

3) Short-term update every time slot Update the vector $\mathbf{q}$ according to (12). Then, compute $\mathbf{p}^{(n+1)}$ based on $\mathbf{q}^{(n+1)}$ using (11). 
Unlike conventional wireless networks that support voice service, there is no need to maintain a constant SINR at all times. The proposed optimal power allocation is a decreasing function of its effective interference. In addition, the maximum power is bounded by $1 / \lambda$. Thus, MT-NPG will never result in powers "blowing up", even in a system with an infinite number of nodes. However, with the number of nodes increasing, condition (13) may not hold anymore. In this work we focus only on systems that satisfies (13), for which an MT-NPG system has the following properties:

1) Because the power allocation is bounded, a fixed point exists.

2) The fixed point is unique.

3) The power vector $\mathbf{p}$ converges to the fixed point $\mathbf{p}^{*}$.

4) If the initial power is the maximal power, the sequence of power vector generated by MT-NPG is monotonically decreasing and converges to $\mathbf{p}^{*}$; If it is zero vector, the sequence converges to $\mathbf{p}^{*}$ increasingly.

5) For all $i=1,2, \ldots, N$, the larger the value of $\lambda_{i}$, the smaller the value of $p_{i}^{*}$.

The properties can be proven by applying the theorems in [11].

\section{NUMERICAL RESUltS}

In this section, we evaluate the performance of MT-NPG using simulations. First, we consider a two-link network to demonstrate the iterative procedure and the effect of the parameter $\lambda_{i}$ on the performance. We set the effective link gain $g_{11}=g_{22}=1, g_{12}=g_{21}=0.25$ and the noise powers $\eta_{1}=\eta_{2}=0.5$. In Fig. 2 , the curve of $p_{2}=F_{2}\left(p_{1}\right)$ is the iterative function of $p_{2}$ with respect to $p_{1}$. Correspondingly, $p_{1}=F_{1}\left(p_{2}\right)$ is the trajectory along which $p_{1}$ is updated according to $p_{2}$. When the initial power is the maximum power, the sequence of power vector is generated along the direction of arrow until it reach the Nash equilibrium. The Nash equilibrium the point $(0.56,0.56)$ is achieved when $\lambda_{1}=\lambda_{2}=1$, and the point $(0.68,0.12)$ when $\lambda_{1}=1, \lambda_{2}=2$.

The value of $\lambda_{i}$ influences the position of the Nash equilibrium. The long-term power consumption can be controlled by adjusting the parameter $\lambda_{i}$. When the power consumption is higher than the average power constraint, we can increase $\lambda_{i}$, and vice versa.

Considering a three-link network, we assume the effective link gain $g_{11}, g_{22}, g_{33}$ are fixed and equal to $1,0.5$, and 0.2 , respectively, and $g_{i j}$ is 0.1 for $i \neq j$. The AWGN power is 0.05 , and $\lambda_{1}=\lambda_{2}=\lambda_{3}=1$. Fig. 3 shows the evolution of power convergence using the MT-NPG algorithm. It is seen that, the powers converge to $0.80,0.58$ and 0.03 , respectively. The corresponding SINR's are 7.2, 2.2, 0.03. The total throughput is $2.78 \mathrm{Nats} / \mathrm{s} / \mathrm{Hz}$, which is 1.58 times more than the one using Foschini-Miljanic algorithm [15], even though the total power consumed by MT-NPG is much lower. MT-NPG can be seen as a type of opportunistic communications. When the effective interference is low, the node increases the transmission power. On the contrary, Foschini-Miljanic algorithm is to increase the transmission power when the effective interference is high in order to guarantee his target SINR.

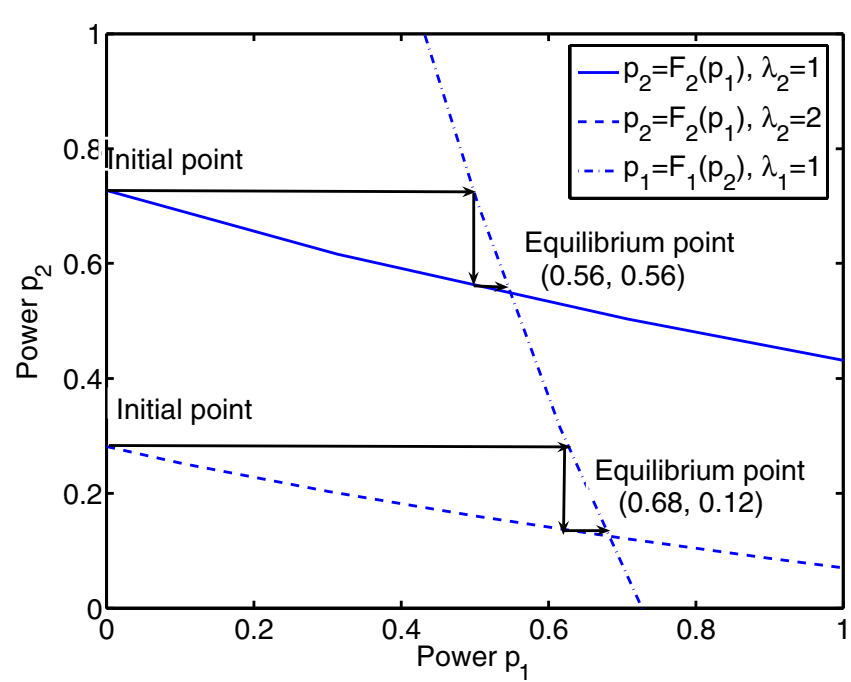

Fig. 2. Illustration of iterative procedure and the effect of parameter $\lambda$ on the performance
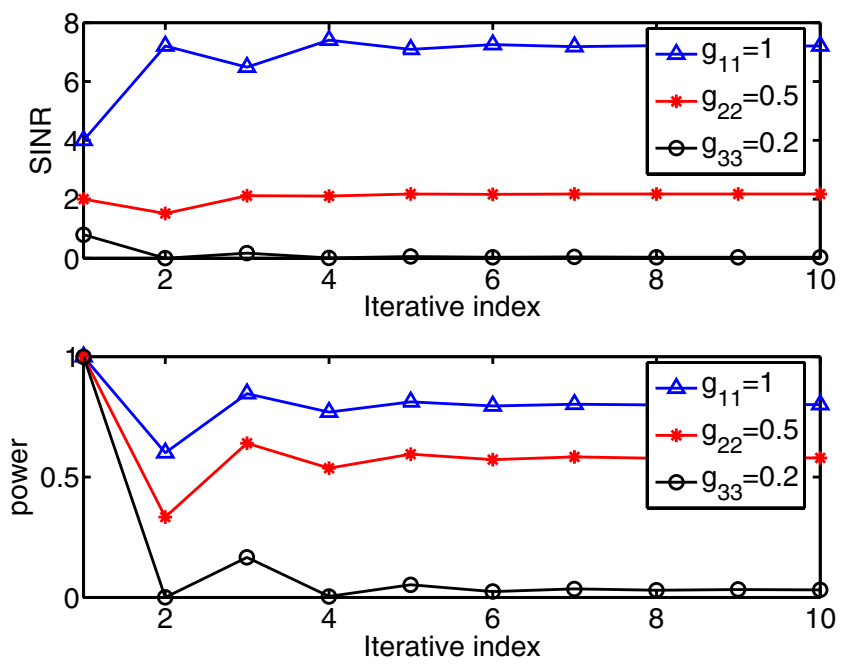

Fig. 3. Evolution of SINR and power using MT-NPG algorithm when the $\lambda_{i}=1$ for $i=1,2,3$.

Fig. 4 shows the effect of $\lambda$ on the Nash equilibrium. We set all the link gains equal 0.5 . It is seen that the power converges to a smaller value when the associated $\lambda$ increases.

Finally, we compare the system performance of the proposed MT-NPG with that of Sung's opportunistic power algorithm (SOPA). The principle of SOPA is to maintain a constant signal-interference product, $\boldsymbol{\alpha}$. The associated iterative function is given as

$$
\mathbf{F}_{\mathbf{p}}=\frac{\alpha}{\mathbf{I}(\mathbf{p})}
$$

We simulate a network in a $10 \mathrm{~m} \times 10 \mathrm{~m}$ square area with 3 , 6, and 9 links, respectively. Transmitters and receivers are randomly placed in this area following a uniform distribution. We assume that the path loss exponent is 4 with no shadowing. The cross correlation between each node is 0.01 . Each link 

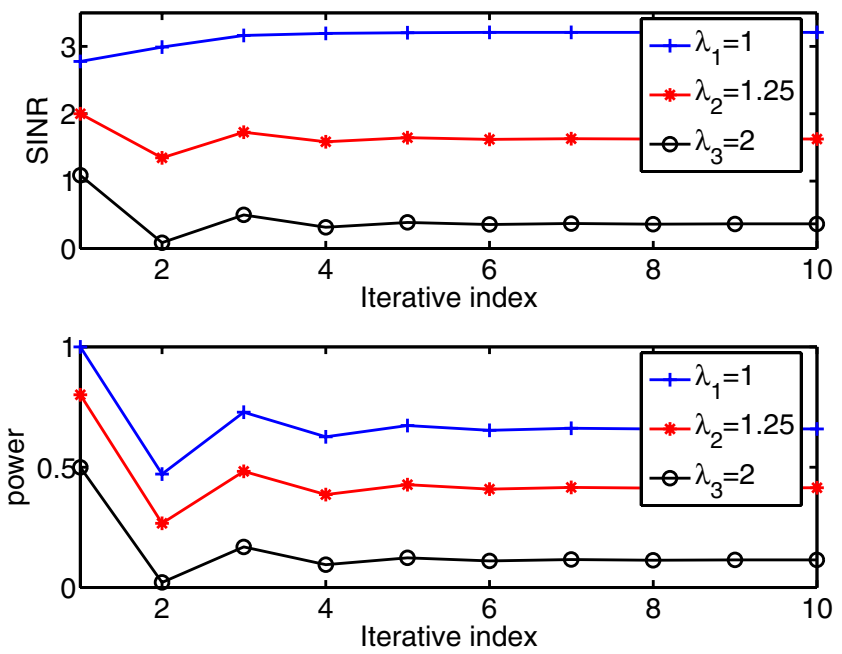

Fig. 4. Evolution of SINR and power using MT-NPG algorithm when $g_{i i}=$ 0.5 for $i=1,2,3$.

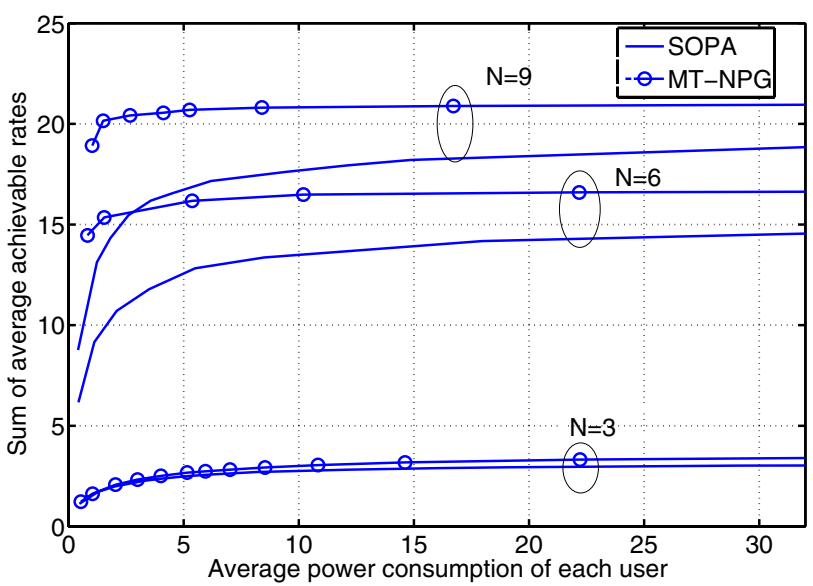

Fig. 5. Sum of average rates vs average power for SOPA and MT-NPG

suffers from Rayleigh fading. The noise power $\eta_{i}$ is set to be 0.1 . We generate $\mathrm{L}=15000$ scenarios randomly. For each scenario, the power is iterated for 50 times. Fig. 5 compares the sum of average throughputs against average power consumption of each user for the SOPA and MT-NPG. For SOPA, the curve is obtained by adjusting the target signalinterference product $\boldsymbol{\alpha}$. The larger the quantity of $\boldsymbol{\alpha}$ is, the more power is consumed. For MT-NPG, the average power constraint is obtained by adjusting $\lambda$. The larger the value of $\lambda$ is, the less power is consumed. One can observe that the proposed MT-NPG has a throughput gain over SOPA ranging from $13 \%$ to $50 \%$.

\section{CONCLUSION}

In this paper, we study non-cooperative power control in the Rayleigh-faded wireless network. We assume that the best response of a node to the actions taken by others is to adjust its power so as to maximize the average achievable rate. It is shown that the optimal power is a monotonically decreasing function of the effective interference. In addition, the optimal power is bounded. An MT-NPG is developed by constructing a novel iterative function. Numerical results show that our proposed MT-NPG achieves a higher throughput than existing algorithms given the same power constraint.

\section{APPENDIX I \\ PROOF OF TYPE II STANDARD}

Proof: It is obvious that $\mathbf{J}$ satisfies monotonicity. It remains to prove that $\mathbf{J}$ satisfies scalability. Rearranging (9), for any $i=1,2, \ldots, N$, we have

$$
J_{i}(\mathbf{q})=\frac{2}{\lambda^{2}} \frac{1}{\left(\sum_{j \neq i} \frac{g_{i j}}{g_{i i}} q_{j}\right)+\frac{\eta_{i}}{g_{i i}}+\frac{1}{\lambda_{i}}-\sum_{j \neq i} \frac{g_{i j}}{g_{i i}} \frac{1}{\lambda_{j}}}
$$

It can be observed from the above equation that, if

$$
\frac{\eta_{i}}{g_{i i}}+\frac{1}{\lambda_{i}}-\sum_{j \neq i} \frac{g_{i j}}{g_{i i} \lambda_{l}}>0
$$

then $J_{i}(\alpha \mathbf{q})>\frac{1}{\alpha} J_{i}(\mathbf{q})$ for all $\alpha>1$. Therefore, the iterative function $\mathbf{J}$ is type II standard if (14) is satisfied.

\section{REFERENCES}

[1] J. Zander, "Performance of optimum transmitter power control in cellular radio systems," IEEE Trans. Veh. Tech., vol. 41, no. 1, Feb. 1992.

[2] L. Li, N. Jindal, and A. J. Goldsmith, "Outage capacities and optimal power allocation for fading multiple-access channels," IEEE Trans. on Infor. Theory, vol. 51, no. 4, pp. 1326-1347, April 2005.

[3] A. J. Goldsmith and S. B. Wicker, "Design challenges for energyconstrained ad hoc wireless networks," IEEE wirel. commun. Mag., vol. 9, no. 4, pp. 8-27, Aug. 2002.

[4] A. Goldsmith and P. Varaiya, "Capacity of fading channels with channel side information," IEEE Transa. on Infor. Theory, vol. 43, no. 11, pp. 1986-1992, Nov. 1997.

[5] P. Viswanath, D. Tse, and R. Laroia, "Opportunistic beamforming using dumb antennas," IEEE Trans. on Info. Theory, vol. 48, no. 6, pp. 12771294, June 2002.

[6] R. Knopp and P. A. Humblet, "Information capacity and power control in single-cell multiuser communications," in Proc. IEEE Int. Conf. Commun. (ICC), 1995

[7] Z. Han and K. J. R. Liu, "Noncooperative power-control game and throughput game over wireless networks," IEEE Trans. on Commun., vol. 53, no. 10 , pp. $1625-1629$, Oct. 2005

[8] C. U. Saraydar, N. B. Mandayam, and D. J. Goodman, "Efficient power control via pricing in wireless data networks," IEEE on Trans. Commun., vol. 50, no. 2, pp. 291-303, Feb. 2002.

[9] N. Feng, S. C. Mau, and N. B. Mandayam, "Pricing and power control for joint user-centric and network-centric resource allocation," IEEE Trans. on Commun., vol. 52, no. 9, pp. 1547-1557, Sep. 2004.

[10] C. W. Sung, K. W. Shum, and K. K. Leung, "Stability of distributed power and signature sequence control for cdma systems - a game theoretic approach," IEEE Trans. on Infor. Theory, vol. 52, no. 4, pp. 1775-1780, April 2006.

[11] C. W. Sung and K. K. Leung, "A generalized framework for distributed power control in wireless networks," IEEE Trans. on Infor. Theory, vol. 51, no. 7, pp. 2625-2635, June 2005.

[12] B. S. Gottfried and J. Weisman, Introduction to optimization theory. Prentice-Hall, 1973.

[13] S. Dey and J. S. Evans, "Optimal power control over multiple timescale fading channels with service outage constraints," IEEE Trans. on Commun., vol. 53, no. 4, pp. 708-717, April 2005.

[14] R. D. Yates, "A framework for uplink power control in cellular radio systems," IEEE J. Sel. Areas Commun., vol. 13, no. 7, pp. 1341-1347, Sep. 1995.

[15] G. Foschini and Z. Miljanic, "A simple distributed autonomous power control algorithm and its convergence," IEEE Trans. Veh. Tech., vol. 42, no. 4, pp. 641-646, Nov. 1993. 